\title{
Clinical Application of 3D Arterial Spin-Labeled Brain Perfusion Imaging for Alzheimer Disease: Comparison with Brain Perfusion SPECT
}

\author{
H. Takahashi, K. Ishii, C. Hosokawa, T. Hyodo, N. Kashiwagi, M. Matsuki, R. Ashikaga, and T. Murakami
}

\begin{abstract}
BACKGROUND AND PURPOSE: Alzheimer disease is the most common neurodegenerative disorder with dementia, and a practical and economic biomarker for diagnosis of Alzheimer disease is needed. Three-dimensional arterial spin-labeling, with its high signal-to-noise ratio, enables measurement of cerebral blood flow precisely without any extrinsic tracers. We evaluated the performance of 3D arterial spin-labeling compared with SPECT, and demonstrated the 3D arterial spin-labeled imaging characteristics in the diagnosis of Alzheimer disease.
\end{abstract}

MATERIALS AND METHODS: This study included 68 patients with clinically suspected Alzheimer disease who underwent both 3D arterial spin-labeling and SPECT imaging. Two readers independently assessed both images. Kendall W coefficients of concordance $(K)$ were computed, and receiver operating characteristic analyses were performed for each reader. The differences between the images in regional perfusion distribution were evaluated by means of statistical parametric mapping, and the incidence of hypoperfusion of the cerebral watershed area, referred to as "borderzone sign" in the 3D arterial spin-labeled images, was determined.

RESULTS: Readers showed $K=0.82 / 0.73$ for SPECT/3D arterial spin-labeled imaging, and the respective areas under the receiver operating characteristic curve were $0.82 / 0.69$ for reader 1 and $0.80 / 0.69$ for reader 2 . Statistical parametric mapping showed that the perisylvian and medial parieto-occipital perfusion in the arterial spin-labeled images was significantly higher than that in the SPECT images. Borderzone sign was observed on 3D arterial spin-labeling in $70 \%$ of patients misdiagnosed with Alzheimer disease.

CONCLUSIONS: The diagnostic performance of 3D arterial spin-labeling and SPECT for Alzheimer disease was almost equivalent. Threedimensional arterial spin-labeled imaging was more influenced by hemodynamic factors than was SPECT imaging.

ABBREVIATIONS: $\quad$ AD = Alzheimer disease; $\mathrm{ASL}=$ arterial spin-labeling; $\mathrm{ROC}=$ receiver operating characteristic; $\mathrm{HMPAO}=$ hexamethylpropyleneamine oxime

A lzheimer disease $(\mathrm{AD})$ is the most common neurodegenerative disorder with dementia and is becoming a social problem in most developed countries. A practical and economic biomarker for diagnosis of $\mathrm{AD}$ is needed. CBF is commonly accepted as a physiologic correlate of brain function. ${ }^{1} \mathrm{AD}$ is associated with regional decreases in $\mathrm{CBF}$, so the ability of $\mathrm{CBF}$ to differentiate between individuals affected by $\mathrm{AD}$ and healthy individuals has been evaluated with the use of SPECT. ${ }^{2}$

Arterial spin-labeling (ASL) enables measurement of $\mathrm{CBF}$ without any extrinsic tracers by use of magnetically labeled arterial blood water as a diffusible tracer. ASL MR imaging has 2

Received July 8, 2013; accepted after revision September 10.

From the Department of Radiology, Kinki University Faculty of Medicine, OsakaSayama, Japan

Please address correspondence to $\mathrm{H}$. Takahashi, MD, Kinki University Faculty of Medicine 377-2 Ohno-Higashi, Osaka-Sayama, Japan; e-mail: hiroto.takahashi07@ gmail.com

http://dx.doi.org/10.3174/ajnr.A3780 major modalities: pulsed $\mathrm{ASL}^{3}$ and continuous ASL. ${ }^{4}$ The continuous ASL technique uses continuous adiabatic inversion, whereas pulsed ASL uses a single inversion pulse. The recently developed pulsed-continuous ASL imaging protocol based on 3D stack-ofspirals readouts ${ }^{5}$ is an intermediate method between the conventional pulsed ASL and continuous ASL methods, in that pulsedcontinuous offers a longer tag bolus than does pulsed ASL and a higher labeling efficiency than does the amplitude-modulated continuous ASL. ${ }^{6,7}$ Each section acquired with 2D ASL experiences a slightly different inflow time; thus, it is difficult to estimate a precise transit time when multiple sections are acquired. The use of $3 \mathrm{D}$ acquisition techniques overcomes many of these limitations, allowing both whole-brain coverage and simultaneous acquisition to ensure a unified mean transit time. The SNR of 3D acquisitions can be greater than that of $2 \mathrm{D}$ multisection methods. Although ASL has inherently low SNR, mainly because of the relatively small amount of labeled spins in the tissue, pulsed-continuous can provide a better balance between labeling efficiency 
and SNR than conventional ASL methods. This can improve the accuracy of quantified CBF estimates. Many AD studies by use of ASL have been reported, which indicates that ASL MR imaging is an indispensable technique for studying $\mathrm{AD} .{ }^{8-12} \mathrm{CBF}$ measured with ASL MR imaging can detect regional hypoperfusion in the $\mathrm{AD}$ precuneus and bilateral parietal cortex and discriminate individuals with $\mathrm{AD}$ from normal subjects. Recent research with the use of pulsed-continuous reported that 3D ASL can evaluate the severity of cognitive impairment as measured by the correlation of CBF with cognition. ${ }^{13}$

SPECT is now commonly used for CBF assessment in the diagnosis of $\mathrm{AD}$, so we considered that it was important to evaluate the differences in CBF distribution in perfusion images obtained with both SPECT and ASL by use of similarly behaved diffusible tracers and to demonstrate the characteristics of ASL in comparison with SPECT. To the best of our knowledge, the evaluation of brain perfusion imaging by use of both ASL and SPECT in the same subjects with clinically suspected $\mathrm{AD}$ to discriminate the $\mathrm{AD}$ group from the non-AD group has not been reported. We used whole-brain 3D ASL MR imaging with pulsed-continuous labeling for $\mathrm{CBF}$ measurement in the diagnosis of $\mathrm{AD}$ because of its high SNR and the possibilities for improving image quality.

In this study, we evaluated the detectability of reduced regional cerebral perfusion in AD by use of 3D ASL compared with brain perfusion SPECT and demonstrated the characteristics of perfusion images obtained by means of 3D ASL.

\section{MATERIALS AND METHODS}

Our institutional review board approved this study. The requirement for participant informed consent was waived because of the retrospective nature of the study.

\section{Patients}

We retrospectively selected 68 consecutive patients with clinically suspected AD from the period between May 2011 and December 2012. Patients included 21 men and 47 women (age range: 53-93 years; mean, 77 years). All patients received the Japanese version of the Mini-Mental State Examination, ${ }^{14}$ and their scores ranged from 6-29 points (mean, 21 points). MR examinations were performed as part of the patients' routine clinical care. All patients underwent brain perfusion imaging both with 3D ASL on a 1.5T clinical MR scanner and with technetium Tc99m-hexamethylpropylene amine oxime (HMPAO) SPECT within a 1-month period. Structural MR imaging was performed in addition to brain perfusion imaging to confirm that there was no other structural abnormality that could explain the patient's symptoms. Patients had no history of cerebrovascular disease, brain tumor, head trauma, or other causes of dementia. Thirty-six patients were diagnosed with $\mathrm{AD}$ and 32 patients were diagnosed as non-AD by experienced neurologists and psychiatrists, by use of the criteria of the National Institute of Neurologic and Communicative Disorders and Stroke in concert with the Alzheimer Disease and Related Disorders Association, ${ }^{15}$ and the Diagnostic and Statistical Manual of Mental Disorders, 4th edition. ${ }^{16}$ Four patients with mild cognitive impairment who later converted to $\mathrm{AD}$ were included in the $\mathrm{AD}$ group. Among patients diagnosed as non-AD, 5 patients had dementia with Lewy bodies, 3 patients had vascular dementia,
Table 1: Demographic characteristics of the subjects

\begin{tabular}{lll}
\hline & \multicolumn{1}{c}{ Patients with AD } & Patients with Non-AD \\
\hline Numbers & 36 & 32 \\
Age, $y$ & Mean, 78 (range: 55-93) & Mean, 72 (range: 53-92) \\
Sex: male:female & $9: 27$ & $12: 20$ \\
MMSE scores & Mean, 19 (range: 6-26) & Mean, 23 (range: 13-29) \\
Included patients & $\mathrm{AD}=32 ; \mathrm{MCl}$ due & $\mathrm{DLB}=5 ; \mathrm{VaD}=3 ;$ \\
& to $\mathrm{AD}=4$ & $\mathrm{CN}=24$ \\
\hline
\end{tabular}

Note:-MMSE indicates Mini-Mental State Examination; DLB, dementia with Lewy bodies; $\mathrm{VaD}$, vascular dementia; $\mathrm{CN}$, cognitively normal.

and 24 were cognitively normal. The diagnosis of dementia with Lewy bodies was based on the criteria of the DLB Consortium. ${ }^{17}$ Vascular dementia was diagnosed according to the criteria of the National Institute of Neurologic Disorders and Stroke-Association Internationale pour la Recherche et l'Enseignement en Neuroscience. ${ }^{18}$ A summary of the demographic characteristics and neuropsychological test results for the patients is shown in Table 1.

\section{MR Imaging}

All MR imaging was performed with a $1.5 \mathrm{~T}$ unit (Signa; GE Healthcare, Milwaukee, Wisconsin) and an 8-channel head array receiving coil. Pulsed-continuous imaging (3D fast spin-echo acquisition with background suppression, a labeling period of 1450 $\mathrm{ms}$, postlabeling delay of $1525 \mathrm{~ms}$, echo time of $10.47 \mathrm{~ms}$, repetition time of $4546 \mathrm{~ms}$, spiral readout of 8 arms $\times 512$ samples, $30 \times 4.0-\mathrm{mm}$ axial sections, $3.2 \times 3.2-\mathrm{mm}$ in-plane resolution, reconstructed pixel size of $1.7 \times 1.7 \mathrm{~mm}$, acquisition time of 4 minutes, 30 seconds) was performed with the labeling plane at the level of the foramen magnum. Labeled and reference images were then subtracted to obtain perfusion-weighted imaging data. In addition to ASL imaging, all patients underwent axial diffusionweighted (repetition time of $6000 \mathrm{~ms}$, echo time of $70 \mathrm{~ms}$, b factor of $1000 \mathrm{~ms} / \mathrm{mm}^{2}, 3$ directions) and axial T2-weighted fast spinecho (repetition time of $4717 \mathrm{~ms}$, echo time of $85 \mathrm{~ms}$ ) imaging to confirm that there was no other structural abnormality. All of these examinations were performed with a $5-\mathrm{mm}$ section thickness, 1.5-mm skip, and 24-cm field of view. In addition, highresolution 3D T1-weighted whole-brain images (repetition time of $8.3 \mathrm{~ms}$, echo time of $3.8 \mathrm{~ms}$, inversion time of $240 \mathrm{~ms}, 8^{\circ}$ flip angle, matrix of $256 \times 256,176$ sections, voxel size of $1.0 \times 0.9 \times$ $0.9 \mathrm{~mm}$, imaging time of 4 minutes, 50 seconds) were obtained by use of the magnetization-prepared rapid acquistion of gradient echo (spoiled gradient-echo) sequence for anatomic information. The images were obtained in sagittal planes and were reconstructed into 1-mm-thick consecutive transverse images.

\section{SPECT}

Patients were directed to lie down for 20 minutes in the supine position with their eyes closed in a dim, quiet room. SPECT was performed by use of a double-head gamma camera (Forte; ADAC Laboratories, Milpitas, California) equipped with a Vertex general purpose collimator (VXGP; ADAC Laboratories) and converging collimators with a focal length of $65 \mathrm{~cm}$. The acquisition began 7 minutes after the injection of $740 \mathrm{MBq}$ of HMPAO, and projection data were obtained at 25 seconds/step $\times 32$ views, resulting in a total acquisition time of approximately 20 minutes. A Butterworth filter (cutoff: 0.25, order: 8) was used for filtered back-projection reconstruction of the SPECT image, yielding a 

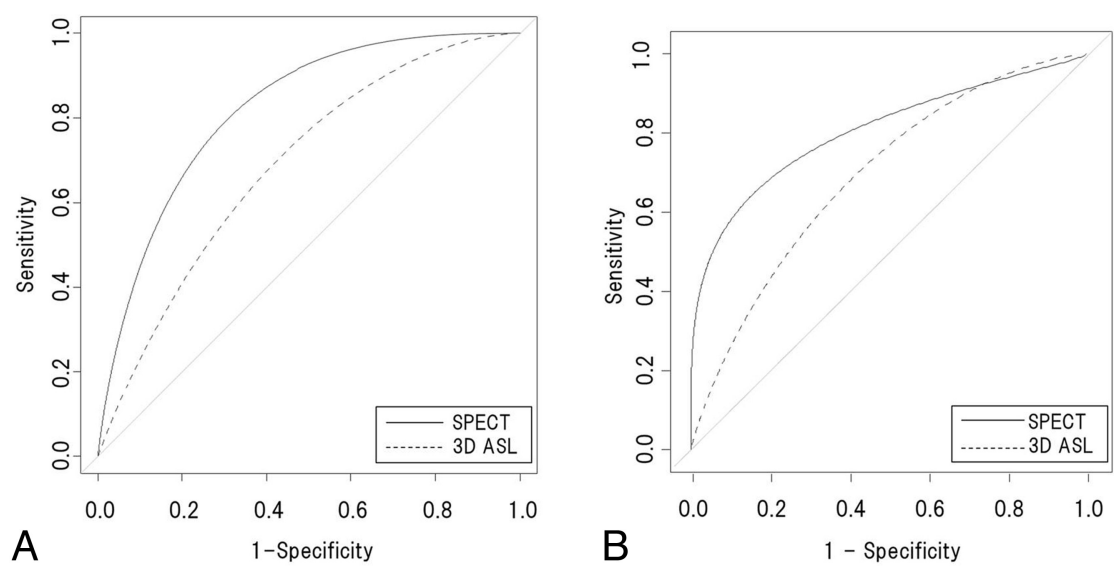

the 2 readers. A confidence level of 4 or 5 was considered a positive finding for the calculation of sensitivity and specificity of $\mathrm{AD}$ diagnosis, but the readers were not informed of this. The area under the ROC curve, sensitivity, and specificity were statistically compared between the readers by use of the bootstrap test for 2 correlated ROC curves. In addition, a paired $t$ test was performed to evaluate the differences in regional perfusion distribution between the 2 imaging methods, by use of voxel-based analysis with statistical parametric mapping. Both 3D ASL and SPECT image preprocessing were implemented by use of SPM 8 software (Wellcome Department of Imaging Neuroscience, London, United Kingdom) and code written in

reconstructed pixel size of $2.5 \mathrm{~mm}$ within the transverse plane and a section thickness of $3.6 \mathrm{~mm}$; attenuation correction was performed by use of the Chang method (JETStream Workspace 3.0. Philips Healthcare, Best, The Netherlands). No scatter compensation was available on the SPECT system.

\section{Image Analysis}

The ASL and SPECT data were evaluated with visual inspection and 3D stereotactic surface projection $z$ score maps. The normal control data base for 3D stereotactic surface projection of ASL perfusion images was constructed with the newly prepared ASL images of 30 cognitively normal subjects: 11 men and 19 women (age range: 64-84 years; mean age, 73 years) who underwent brain MR imaging because of indefinite complaints and who had no structural abnormalities such as cerebrovascular disease, brain tumor, head trauma, or neurodegenerative disorder. The preset normal data base for HMPAO SPECT in the iSSP program (Nihon Medi-Physics, Tokyo, Japan) was used for this study. Two readers (C.H., K.I.) other than the study coordinator independently reviewed the SPECT images and 3D ASL images. These 2 readers were radiologists with 22 and 25 years of experience in nuclear medicine and were blinded to the results of the examination for AD. In both SPECT images and 3D ASL images, they assessed focal hypoperfusion in areas over the bilateral precunei and posterior cingulate gyri. They recorded their confidence levels regarding the presence of focal hypoperfusion as an indicator of $\mathrm{AD}$ by use of the following 5-point rating system: 1 , definitely absent; 2 , probably absent; 3 , presence equivocal; 4 , probably present; 5 , definitely present. Kendall W coefficients of concordance $(K)$ were computed to compare the assessment of the level of confidence of the 2 readers. Kendall W coefficients $(K)$ of $0.5-0.8$ were considered to indicate good agreement, and coefficients higher than 0.8 were considered to indicate excellent agreement. A value of $P<.05$ was considered to indicate a significant difference. Receiver operating characteristic (ROC) analysis to compare the diagnostic performance was performed with the aid of R software (Version 2.12.1; http://www.r-project.org/). ${ }^{19}$ The area under the ROC curve was calculated to compare the diagnostic performance for both the 3D ASL images and SPECT images for each of
Matlab (MathWorks, Natick, Massachusetts). For each subject, images were preprocessed as follows: 1) individual 3D ASL images were coregistered with individual SPECT images; 2 ) the SPECT images were spatially normalized to the Montreal Neurological Institute space by the SPM program; 3) the 3D ASL images were then spatially normalized to the Montreal Neurological Institute space by using the individual parameter obtained from SPECT normalization; 4) the spatially normalized images were smoothed by use of an isotropic Gaussian kernel with a 12-mm full width for 3D ASL and $8 \mathrm{~mm}$ for SPECT images at half maximum to reduce the noise and residual anatomic differences among the brains and to match the spatial resolution of 3D ASL and SPECT images; and 5 ) a paired $t$ test was performed by using both preprocessed $3 \mathrm{D}$ ASL and SPECT images. To control for family-wise errors resulting from multiple comparisons, the initial significance threshold was set at $P<.001$.

In a recent report, hypoperfusion of the watershed area on $3 \mathrm{D}$ ASL imaging in many healthy subjects was referred to as the "borderzone sign." ${ }^{20}$ We considered that this sign might have an effect on the performance of $\mathrm{AD}$ diagnosis; therefore we evaluated the frequency of this sign in the diagnostic discrepancy group. The patients with a discrepancy between clinical diagnosis and 3D ASL results and with a diagnostic discrepancy for reader 1 by use of SPECT-3D ASL were assessed. The borderzone sign was evaluated on the simple criterion that the signal defect of the watershed area in the 3D ASL images was positive by consensus between the 2 readers (C.H., K.I.).

\section{RESULTS}

The 2 readers showed excellent agreement for the diagnosis of $\mathrm{AD}$ on the SPECT images $(K=0.82, P=.00087)$ and good agreement for that on the 3D ASL images $(K=0.73, P=.0083)$. The ROC curves for 3D ASL and SPECT images obtained by each reader are shown in Fig $1 A,-B$. For reader 1 , the area under the ROC curve distinguishing $\mathrm{AD}$ from non-AD was 0.82 for SPECT images alone and 0.69 for $3 \mathrm{D}$ ASL images alone. A bootstrap test for the 2 correlated ROC curves showed that there was no significant difference $(P=.08)$ between the 2 images. For reader 2 , the area under the ROC curve distinguishing 
3D ASL - SPECT
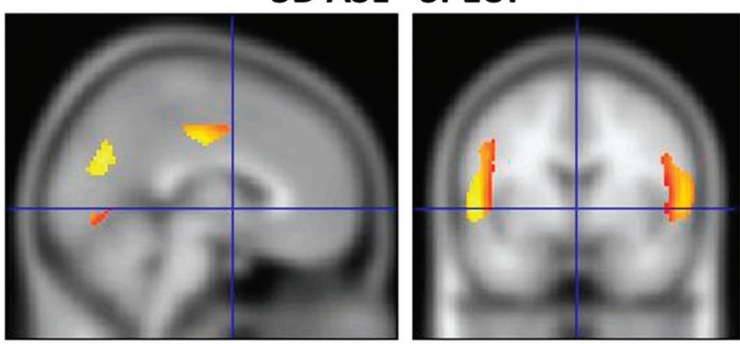

A
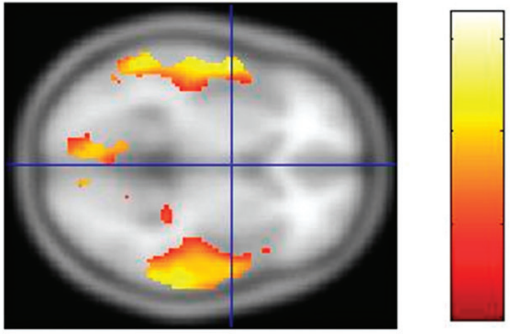

FIG 2. SPM analysis revealed that the perfusion images of 3D ASL were delineated by areas of higher CBF (red-to-yellow areas) in the middle cerebral artery region and in the posterior cerebral artery region than those observed on SPECT images ( $A$ ), whereas small areas of higher CBF (red-to-yellow areas) were scattered in the SPECT perfusion images compared with those on 3D ASL (B).

Table 2: Clinical diagnosis and 3D ASL diagnosis

\begin{tabular}{ccc}
\hline & \multicolumn{2}{c}{ 3D ASL } \\
\cline { 2 - 3 } & Correct & Incorrect \\
\hline Patients with $\mathrm{AD}, n=36$ & 17 & 19 \\
Borderzone sign $(+:-)$ & $(6: 11)$ & $(6: 13)$ \\
Patients with non-AD, $n=32$ & 22 & 10 \\
Borderzone sign $(+:-)$ & $(5: 17)$ & $(7: 3)$ \\
\hline
\end{tabular}

Table 3: Clinical diagnosis, SPECT diagnosis, and 3D ASL diagnosis

\begin{tabular}{cccc}
\hline & SPECT/3D ASL & & SPECT/3D ASL \\
\cline { 2 - 2 } & Correct/Incorrect & & Incorrect/Correct \\
\hline Patients with AD, $n=19$ & 14 & 5 \\
Borderzone sign $(+:-)$ & $(4: 10)$ & & $(2: 3)$ \\
Patients with non-AD, $n=9$ & 5 & & 4 \\
Borderzone sign $(+:-)$ & $(4: 1)$ & & $(0: 4)$ \\
\hline
\end{tabular}

$\mathrm{AD}$ from non-AD was 0.80 for SPECT images alone and 0.69 for $3 \mathrm{D}$ ASL images alone. A bootstrap test for the 2 correlated ROC curves showed that there was no significant difference $(P=.20)$ between the 2 images.

SPM analysis showed that in the 3D ASL image, the perisylvian and medial parieto-occipital perfusion was significantly higher than in the SPECT image $(P<.001)$ (Fig $2 A)$. In contrast, the area of hypoperfusion was barely detected in the $3 \mathrm{D}$ ASL image in comparison with the SPECT image $(P<.001)($ Fig $2 B)$.

The results for the frequency of the borderzone sign and the discrepancy between clinical diagnosis and 3D ASL diagnosis are described in Table 2. The borderzone sign was observed in 6 of 17 patients $(35 \%)$ with AD correctly diagnosed by 3D ASL and in 7 of 10 patients $(70 \%)$ with non-AD incorrectly diagnosed by 3D ASL. The results for the frequency of the borderzone sign and the diagnostic discrepancy between SPECT and 3D ASL are described in Table 3. Diagnostic discrepancy between SPECT and 3D ASL was observed for 28 of 68 patients (41\%). In the 9 patients without $\mathrm{AD}, 1$ patient had dementia with Lewy bodies, 1 patient had vascular dementia, and 7 were cognitively normal. More detailed analysis of these results was not performed because the small number of patients precluded statistical analysis. In the patients with SPECT-3D ASL diagnostic discrepancy, the borderzone sign was observed in 2 of 5 patients ( $40 \%$ ) with AD correctly diagnosed by $3 \mathrm{D}$ ASL and in 4 of 5 patients ( $80 \%)$ with non-AD incorrectly diagnosed by 3D ASL. Representative images of the borderzone sign in patients with diagnostic discrepancy are shown in Fig 3.

\section{DISCUSSION}

CBF assessment by SPECT is established as an indispensable clinical tool in the diagnosis of AD. ${ }^{21,22}$ HMPAO SPECT and FDGPET showed hypoperfusion and decreased metabolism in the temporoparietal and posterior cingulate gyrus in $\mathrm{AD}$, which indicates a direct coupling in $\mathrm{AD}$ between these 2 effects. ${ }^{23}$ In addition, a recent study to compare 3D ASL MR imaging with FDGPET in the same subjects showed similar degrees of functional deficits in the affected areas, ${ }^{24}$ which indicates that 3D ASL MR imaging may be a more easily accessible alternative perfusion technique than SPECT in the diagnosis of AD.

Voxelwise comparison of the ASL images can be useful to help discriminate patients with $\mathrm{AD}$ from healthy subjects. ${ }^{12} \mathrm{~A}$ more recent study indicated that CBF maps obtained with 3D ASL can be a marker for disease severity ranging from mild cognitive impairment to advanced $\mathrm{AD} .^{13}$ Although many recent ASL perfusion studies were performed on 3T MR imaging, it has been shown in other studies that ASL with 1.5T MR imaging could detect regional hypoperfusion, mainly in the temporoparietal association cortex and the posterior cingulate gyrus. ${ }^{8,10,25}$ Another study suggested that CBF mapping by ASL may perform better than MR morphologic analysis by using voxel-based morphometry in discriminating patients with $\mathrm{AD}$ from healthy control subjects and that the combination of the 2 methods was more effective than either method alone. ${ }^{26}$ This result may support our proposal for the combination of structural MR imaging and ASL MR perfusion imaging. Three-dimensional ASL acquisition in our study required 4.5 minutes, which is short enough to be included in a clinical protocol. 


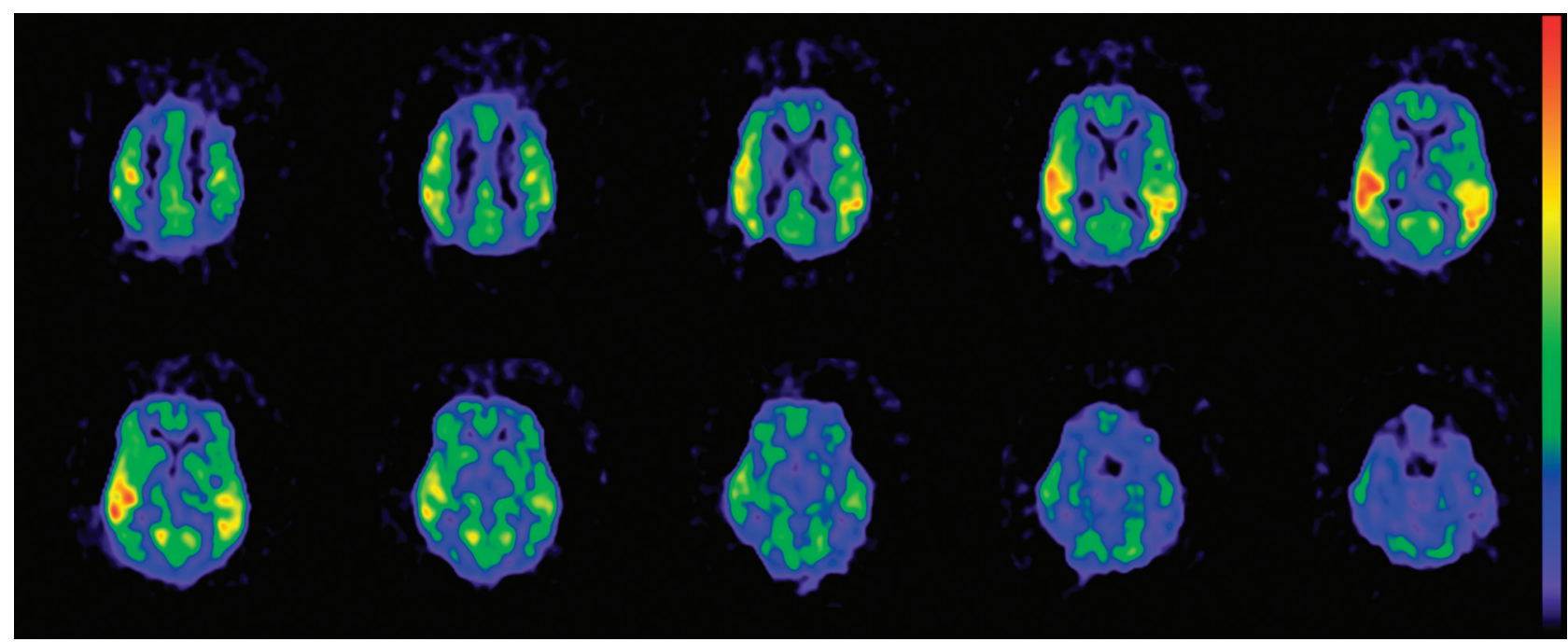

FIG 3. Three-dimensional ASL images frequently show the borderzone sign (watershed area), which indicates bilateral signal deficiency in the middle cerebral artery-anterior cerebral artery and middle cerebral artery-posterior cerebral artery borderzones, with a high signal intensity in the surrounding cortex.

In our study, interobserver agreement for both SPECT and 3D ASL revealed a statistically significant difference (SPECT: $K=$ $0.82, P=.00087$; and 3D ASL: $K=0.73, P=.0083$ ), which may indicate that there were differences between the 2 readers in the accuracy of AD diagnosis. However, interobserver agreement was excellent for the SPECT images and good for the ASL images. In addition, the result of each reader's ROC analysis showed no significant difference between the image types, so we concluded that the diagnostic performance of 3D ASL on a $1.5 \mathrm{~T}$ MR scanner is slightly but not significantly inferior to that of SPECT in the differential diagnosis of $\mathrm{AD}$ and non-AD.

Our results identified regional variability in the reliability of perfusion assessment by ASL. SPM analysis of 3D ASL and SPECT revealed some regional differences in perfusion distribution. In most of the participants, the distribution of perfusion was almost symmetric in the 2 hemispheres. Three-dimensional ASL perfusion images showed higher signal intensities than SPECT images in the MCA region and in the posterior cerebral artery region. We consider that this was because the MCA and posterior cerebral artery have high flow rates and because perfusion images of 3D ASL are influenced more than SPECT by hemodynamics, so the 3D ASL images can identify hypoperfusion of the watershed area. This is associated with aging and is related to a combination of increased arterial transit time and reduced CBF. Diagnostic discrepancies between 3D ASL and SPECT images were observed for 28 patients. We suggest that the misdiagnosis of cases of non- $\mathrm{AD}$ as AD by 3D ASL may result from this effect and that the borderzone sign has major effects on $\mathrm{AD}$ diagnosis as a false-positive finding because of its high incidence ( $70 \%$ and $80 \%)$ in these cases (Tables 2 and 3). Zaharchuk et al ${ }^{20}$ reported that about half of the patients with normal imaging findings on bolus perfusionweighted imaging performed by use of gadolinium enhancement had abnormal ASL findings, most commonly the borderzone sign. The patients included in that study were also older (mean age, $71 \pm 11$ years). These findings suggest that perfusion imaging with 3D ASL is influenced more than SPECT by hemodynamic factors such as arterial transit time, especially in older people. We used a postlabeling delay of $1525 \mathrm{~ms}$, which in previous reports has been suggested to be too short for quantitative CBF measurements in older patients, in whom arterial transit time could potentially be longer. ${ }^{27}$ Our patients with suspected AD were all older (mean, $77 \pm 8$ years). The reason for the use of a postlabeling delay of $1525 \mathrm{~ms}$ is that a longer postlabeling delay causes a decrease in SNR because of T1 relaxation, so there is a trade-off between a longer postlabeling delay and decreased SNR.

SPM analysis revealed the same perfusion of the frontal brain regions on 3D ASL images as on SPECT images, so we believe that the effect of the difference of perfusion distribution between 3D ASL images and SPECT images is of minor importance in evaluation of the function of frontal brain regions (eg, the diagnosis of frontotemporal lobar degeneration). We judged that the result shown in Fig 2B of hypoperfusion on 3D ASL was within the limits of statistical error, because hypoperfusion on the 3D ASL image was visible as small scattered areas and its distribution was not related to cerebral hemodynamics.

Given these limitations, this study can be regarded as a pilot investigation to identify the most suitable ASL images for evaluating CBF distribution, which must be more rigorously examined in future studies. There are 2 factors important in obtaining an accurate CBF map: arterial arrival time and postlabeling delay. In addition, our results indicate that a high magnetic field will be valuable because of its high label efficiency and slower label decay.

\section{CONCLUSIONS}

Perfusion imaging with 3D ASL was influenced to a greater extent than SPECT by hemodynamic factors such as arterial transit time. Statistical analysis revealed that 3D ASL MR imaging can be an alternative perfusion imaging method to SPECT in the diagnosis of $\mathrm{AD}$ and indicates that $3 \mathrm{D}$ ASL is an appropriate method for diagnosing $\mathrm{AD}$ and will allow a "one-stop shop" MR routine examination. 


\section{REFERENCES}

1. Villringer A, Dirnagl U. Coupling of brain activity and cerebral blood flow: basis of functional neuroimaging. Cerebrovasc Brain Metab Rev 1995;7:240-76

2. Soininen H, Helkala EL, Kuikka J, et al. Regional cerebral blood flow measured by $99 \mathrm{mTc}$-HMPAO SPECT differs in subgroups of Alzheimer's disease. J Neural Transm Park Dis Dement Sect 1995; 9:95-109

3. Golay X, Stuber M, Pruessmann KP, et al. Transfer insensitive labeling technique (TILT): application to multislice functional perfusion imaging. J Magn Reson Imaging 1999;9:454-61

4. Alsop DC, Detre JA. Multisection cerebral blood flow MR imaging with continuous arterial spin labeling. Radiology 1998;208:410-16

5. Dai W, Garcia D, de Bazelaire C, et al. Continuous flow-driven inversion for arterial spin labeling using pulsed radio frequency and gradient fields. Magn Reson Med 2008;60:1488-97

6. Wang J, Zhang Y, Wolf RL, et al. Amplitude-modulated continuous arterial spin-labeling 3.0-T perfusion MR imaging with a single coil: feasibility study. Radiology 2005;235:218-28

7. Wu WC, Fernández-Seara M, Detre JA, et al. A theoretical and experimental investigation of the tagging efficiency of pulsed-continuous arterial spin labeling. Magn Reson Med 2007;58:1020-27

8. Alsop DC, Detre JA, Grossman M. Assessment of cerebral blood flow in Alzheimer's disease by spin-labeled magnetic resonance imaging. Ann Neurol 2000;47:93-100

9. Du AT, Jahng GH, Hayasaka S, et al. Hypoperfusion in frontotemporal dementia and Alzheimer disease by arterial spin labeling MRI. Neurology 2006;67:1215-20

10. Johnson NA, Jahng GH, Weiner MW, et al. Pattern of cerebral hypoperfusion in Alzheimer disease and mild cognitive impairment measured with arterial spin-labeling MR imaging: initial experience. Radiology 2005;234:851-59

11. Sandson TA, O'Connor M, Sperling RA, et al. Noninvasive perfusion MRI in Alzheimer's disease: a preliminary report. Neurology 1996;47:1339-42

12. Yoshiura T, Hiwatashi A, Noguchi T, et al. Arterial spin labelling at 3-T MR imaging for detection of individuals with Alzheimer's disease. Eur Radiol 2009;19:2819-25

13. Binnewijzend MA, Kuijer JP, Benedictus MR, et al. Cerebral blood flow measured with 3D pulsed-continuous arterial spin-labeling MR imaging in Alzheimer disease and mild cognitive impairment: a marker for disease severity. Radiology 2013;267:221-30

14. Ideno Y, Takayama M, Hayashi K, et al. Evaluation of a Japanese version of the Mini-Mental State Examination in elderly persons. Geriatr Gerontol Int 2012;12:310-16

15. McKhann G, Drachman D, Folstein M, et al. Clinical diagnosis of Alzheimer's disease: report of the NINCDS-ADRDA Work Group under the auspices of Department of Health and Human Services Task Force on Alzheimer's Disease. Neurology 1984;34:939-44

16. American Psychiatric Association. Diagnostic and Statistical Manual of Mental Disorders: DSM-IV. 4th ed. Washington: American Psychiatric Association; 1994

17. McKeith IG, Dickson DW, Lowe J, et al. Diagnosis and management of dementia with Lewy bodies: third report of the DLB Consortium. Neurology 2005;65:1863-72

18. Román GC, Tatemichi TK, Erkinjuntti T, et al. Vascular dementia: diagnostic criteria for research studies: Report of the NINDSAIREN International Workshop. Neurology 1993;43:250-60

19. R Development Core Team. R: A language and environment for statistical computing. Vienna, Austria: R Foundation for Statistical Computing; 2010

20. Zaharchuk G, Bammer R, Straka M, et al. Arterial spin-label imaging in patients with normal bolus perfusion-weighted MR imaging findings: pilot identification of the borderzone sign. Radiology 2009;252:797-807

21. Johnson KA, Jones K, Holman BL, et al. Preclinical prediction of Alzheimer's disease using SPECT. Neurology 1998;50:1563-71

22. El Fakhri G, Kijewski MF, Albert MS, et al. Quantitative SPECT leads to improved performance in discrimination tasks related to prodromal Alzheimer's disease. J Nucl Med 2004;45:2026-31

23. Herholz K, Schopphoff H, Schmidt M, et al. Direct comparison of spatially normalized PET and SPECT scans in Alzheimer's disease. J Nucl Med 2002;43:21-26

24. Chen Y, Wolk DA, Reddin JS, et al. Voxel-level comparison of arterial spin-labeled perfusion MRI and FDG-PET in Alzheimer disease. Neurology 2011;77:1977-85

25. Asllani I, Habeck C, Scarmeas N, et al. Multivariate and univariate analysis of continuous arterial spin labeling perfusion MRI in Alzheimer's disease. J Cereb Blood Flow Metab 2008;28:725-36

26. Dashjamts T, Yoshiura T, Hiwatashi A, et al. Simultaneous arterial spin labeling cerebral blood flow and morphological assessments for detection of Alzheimer's disease. Acad Radiol 2011;18:1492-99

27. Campbell AM, Beaulieu C. Pulsed arterial spin labeling parameter optimization for an elderly population. J Magn Reson Imaging 2006; 23:398-403 